\title{
Extending Partial Representations of Rectangular Duals with Given Contact Orientations ${ }^{\star}$
}

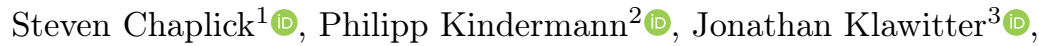 \\ Ignaz Rutter ${ }^{4}\left(\mathbb{D}\right.$, and Alexander Wolff ${ }^{3}$ (]) \\ 1 Maastricht University, Maastricht, The Netherlands \\ ${ }^{2}$ Universität Trier, Trier, Germany \\ 3 Universität Würzburg, Würzburg, Germany \\ 4 Universität Passau, Passau, Germany
}

\begin{abstract}
A rectangular dual of a graph $G$ is a contact representation of $G$ by axis-aligned rectangles such that (i) no four rectangles share a point and (ii) the union of all rectangles is a rectangle. The partial representation extension problem for rectangular duals asks whether a given partial rectangular dual can be extended to a rectangular dual, that is, whether there exists a rectangular dual where some vertices are represented by prescribed rectangles. Combinatorially, a rectangular dual can be described by a regular edge labeling (REL), which determines the orientations of the rectangle contacts.

We describe two approaches to solve the partial representation extension problem for rectangular duals with given REL. On the one hand, we characterise the RELs that admit an extension, which leads to a lineartime testing algorithm. In the affirmative, we can construct an extension in linear time. This partial representation extension problem can also be formulated as a linear program (LP). We use this LP to solve the simultaneous representation problem for the case of rectangular duals when each input graph is given together with a REL.
\end{abstract}

Keywords: rectangular dual · partial representation extension · simultaneous representation

\section{Introduction}

A geometric intersection representation of a graph $G$ is a mapping $\mathcal{R}$ that assigns to each vertex $w$ of $G$ a geometric object $\mathcal{R}(w)$ such that two vertices $u$ and $v$ are adjacent in $G$ if and only if $\mathcal{R}(u)$ and $\mathcal{R}(v)$ intersect. In a contact representation we further require that, for any two vertices $u$ and $v$, the objects $\mathcal{R}(u)$ and $\mathcal{R}(v)$ have disjoint interiors. The recognition problem asks whether a given graph admits an intersection or contact representation whose sets have a specific geometric shape. Classic examples are interval graphs [4], where the

\footnotetext{
* Partially supported by DFG grants Ru 1903/3-1 and Wo 758/11-1. We thank the anonymous reviewers for their helpful comments.
} 
objects are intervals of $\mathbb{R}$, or coin graphs [25], where the objects are interiordisjoint disks in the plane. The partial representation extension problem is a natural generalization of this question where, for each vertex $u$ of a given subset of the vertex set, the geometric object is already prescribed, and the question is whether this partial representation can be extended to a full representation of the input graph. In the last decade the partial representation extension problem has been intensely studied for various classes of intersection graphs, such as (unit or proper) interval graphs [22,23], circle graphs [9], trapezoid graphs [27], as well as for contact representations [8] and bar-visibility representations [10].

A different generalization is the simultaneous representation problem, where, given several input graphs $G_{1}, \ldots, G_{k}$, one asks whether there exist representations $\mathcal{R}_{1}, \ldots, \mathcal{R}_{k}$ of $G_{1}, \ldots, G_{k}$ such that each vertex $v$ contained in $G_{i}$ and in $G_{j}$ satisfies $\mathcal{R}_{i}(v)=\mathcal{R}_{j}(v)$, i.e., any two representations coincide on the shared vertices. Most frequently, this problem is studied in the sunflower case, where one additionally assumes that the pairwise intersection of any two graph $G_{i}, G_{j}$ with $i \neq j$ is the same subgraph $S$, which is usually called the shared graph. The question is equivalent to asking whether there exists a representation of $S$ that simultaneously extends to each of the input graphs $G_{1}, \ldots, G_{k}$. Simultaneous representation problems have long been studied for planar graphs; see $[2,33]$ for surveys. For intersection representations, the problem was originally introduced by Jampani and Lubiw, who gave polynomial-time algorithms for interval graphs [19] as well as for comparability and permutation graphs [20]. They also proved NP-completeness for chordal graphs. Bläsius and Rutter later improved the running time for interval graphs to linear [3]. Recently, Rutter et al. [34] gave efficient algorithms for proper and unit interval graphs. Previous work on simultaneous contact representations has focused on representing planar graphs and their duals, for example, with triangles in the plane [17] or with boxes in 3D [1].

Rectangular duals. In this paper we consider the partial representation extension problem for the following type of representation. A rectangular dual of a graph $G$ is a contact representation $\mathcal{R}$ of $G$ by axis-aligned rectangles such that (i) no four rectangles share a point and (ii) the union of all rectangles is a rectangle; see Fig. 1. We observe that $G$ may admit a rectangular dual only if it is planar and internally triangulated. Furthermore, a rectangular dual can always be augmented with four additional vertices (one on each side) so that only four rectangles touch the outer face of the representation. It is customary that the four vertices on the outer face are denoted by $v_{\mathrm{S}}, v_{\mathrm{W}}, v_{\mathrm{N}}$, and $v_{\mathrm{E}}$ corresponding to the geographic directions, and to require that $\mathcal{R}\left(v_{\mathrm{W}}\right)$ is the leftmost rectangle, $\mathcal{R}\left(v_{\mathrm{E}}\right)$ is rightmost, $\mathcal{R}\left(v_{\mathrm{S}}\right)$ is bottommost, and $\mathcal{R}\left(v_{\mathrm{N}}\right)$ is topmost; see Fig. 1. We call these vertices the outer vertices and the remaining ones the inner vertices. It is known that a plane internally-triangulated graph has a representation with only four rectangles touching the outer face if and only if its outer face is a 4-cycle and it has no separating triangles, that is, a triangle whose removal disconnects the graph [26]. Such a graph is called a properly-triangulated planar (PTP) graph. Kant and He [21] have shown that a rectangular dual of a given PTP graph $G$ can be computed in linear time. 


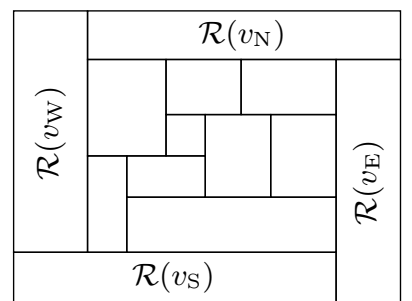

$\mathcal{R}$

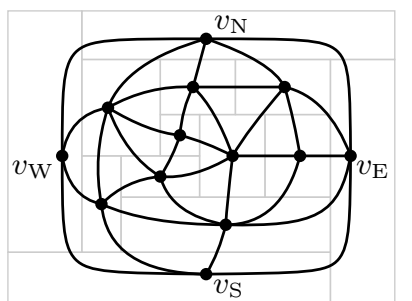

G

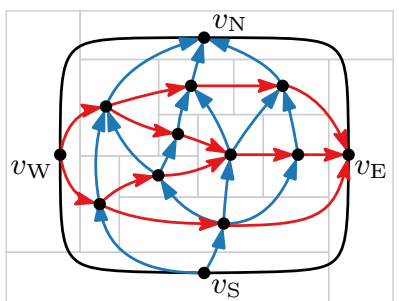

$\left(L_{1}, L_{2}\right)$

Fig. 1. A rectangular dual $\mathcal{R}$ for the graph $G$; the REL $\left(L_{1}, L_{2}\right)$ induced by $\mathcal{R}$.

Historically, rectangular duals have been studied due to their applications in architecture [35], VLSI floor-planning [30,36], and cartography [16]. Besides the question of an efficient construction algorithm [21], other problems concerning rectangular duals are area minimization [7], sliceability [29], and areauniversality, that is, rectangular duals where the rectangles can have any given areas [13]. The latter question highlights the close relation between rectangular duals and rectangular cartograms. Rectangular cartograms were introduced in 1934 by Raisz [32] and combine statistical and geographical information in thematic maps, where geographic regions are represented as rectangles and scaled in proportion to some statistic. There has been lots of work on efficiently computing rectangular cartograms [6,18,28]; Nusrat and Kobourov [31] recently surveyed this topic. As a dissection of a rectangle into smaller rectangles, a rectangular dual is also related to other types of dissections, for example with squares [5] or hexagons [12]; see also Felsner's survey [14].

Regular edge labelings. The combinatorial aspects of a contact representation of a graph $G$ can often be described with a coloring and orientation of the edges of $G$. For example, Schnyder woods describe contact representations of planar graphs by triangles [15]. Such a description also exists for contact representations by rectangles, for example for triangle-free rectangle arrangements [24] or rectangular duals [21]. More precisely, a rectangular dual $\mathcal{R}$ gives rise to a 2-coloring and an orientation of the inner edges of $G$ as follows. We color an edge $\{u, v\}$ blue if the contact between $\mathcal{R}(u)$ and $\mathcal{R}(v)$ is a horizontal line segment, and we color it red otherwise. We orient a blue edge $\{u, v\}$ as $(u, v)$ if $\mathcal{R}(u)$ lies below $\mathcal{R}(v)$, and we orient a red edge $\{u, v\}$ as $(u, v)$ if $\mathcal{R}(u)$ lies to the left of $\mathcal{R}(v)$; see Fig. 1. The resulting coloring and orientation has the following properties:

(1) All inner edges incident to $v_{\mathrm{W}}, v_{\mathrm{S}}, v_{\mathrm{E}}$, and $v_{\mathrm{N}}$ are red outgoing, blue outgoing, red incoming, and blue incoming, respectively.

(2) The edges incident to each inner vertex form four counterclockwise ordered non-empty blocks of red incoming, blue incoming, red outgoing, and blue outgoing, respectively.

A coloring and orientation with these properties is called a regular edge labeling (REL) or transversal structure. We let $\left(L_{1}, L_{2}\right)$ denote a REL, where $L_{1}$ is the 
Chaplick et al.

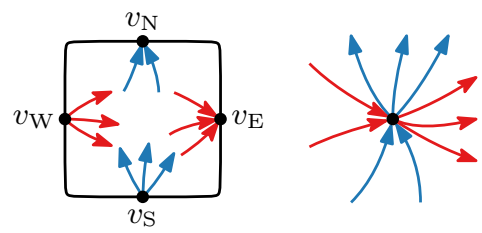

Fig. 2. Edge order at the four outer vertices and an inner vertex.

set of blue edges and $L_{2}$ is the set of red edges. Let $L_{1}(G)$ and $L_{2}(G)$ denote the two subgraphs of $G$ induced by $L_{1}$ and $L_{2}$, respectively. Note that both $L_{1}(G)$ and $L_{2}(G)$ are st-graphs, that is, directed acyclic graphs with exactly one source and exactly one sink. It is well known that every PTP graph admits a REL and thus a rectangular dual [21]. A rectangular dual $\mathcal{R}$ realizes a REL $\left(L_{1}, L_{2}\right)$ if the REL induced by $\mathcal{R}$ is $\left(L_{1}, L_{2}\right)$. Note that while a rectangular dual uniquely defines a REL, there exist different rectangular duals that realize any given REL.

Kant and He [21] introduced RELs and described two linear-time algorithms that compute a REL for a given PTP graph; one algorithm is based on edge contractions, the other is based on canonical orderings. They then use the REL to construct in linear time a rectangular dual that realizes this REL and where the coordinates are all integers.

Partial rectangular duals. For a graph $G$, let $E(G)$ denote the set of edges and $V(G)$ the set of vertices of $G$. Let $U \subseteq V(G)$. Then $G[U]$ denotes the subgraph of $G$ induced by $U$. The pair $(U, \mathcal{P})$ is a partial rectangular dual of $G$ if $\mathcal{P}$ is a contact representation of $G[U]$ that maps each $u \in U$ to an axis-aligned rectangle $\mathcal{P}(u)$. We call the vertices in $U$ fixed and for $u \in U$ we call $\mathcal{P}(u)$ a fixed rectangle. We further define $|\mathcal{P}|=|U|$. For the sake of readability, we refer from now on to a partial rectangular dual $(U, \mathcal{P})$ simply with $\mathcal{P}$ and consider the domain $U$ of $\mathcal{P}$ as implicitly given.

For a given graph $G$ and a partial rectangular dual $\mathcal{P}$, the partial rectangular dual extension problem asks whether $\mathcal{P}$ can be extended to a rectangular dual $\mathcal{R}$ of $G$. In particular, for such an extension $\mathcal{R}$ and each fixed vertex $u$, we require that $\mathcal{P}(u)=\mathcal{R}(u)$. In this paper, we study the variant of this problem where we are not only given $G$ and $\mathcal{P}$, but also a REL $\left(L_{1}, L_{2}\right)$ of $G$ and ask whether there is an extension $\mathcal{R}$ of $\mathcal{P}$ that realizes $\left(L_{1}, L_{2}\right)$.

Closely related work includes partial representation extension of segment contact graphs [8] and bar-visibility representations [10]. Both problems are NPcomplete. However, the hardness reductions crucially rely on low connectivity for choices in the planar embedding. Since PTP graphs are triconnected, they have a unique planar embedding and hence these results cannot be easily transferred.

Contribution and outline. Our first contribution is a linear program (LP) in the form of a system of difference constraints to compute rectangular duals for PTP graphs with given RELs. We show how to use the LP (i) to construct a rectangular dual, (ii) to solve the partial representation extension problem, and 
(iii) to solve the respective simultaneous representation problem in quadratic time; see Section 2.

We then give a characterization of RELs that admit an extension of a given partial rectangular dual via the existence of what we will call a boundary path set; see Section 3. Next, we provide an algorithm that constructs a boundary path set (if possible) as well as an algorithm that computes a representation extension from a boundary path set. Both algorithms run in $\mathcal{O}(n h)$ time, where $n=|V(G)|$ and $h=|\mathcal{P}|$, and are detailed in Section 4. Finally, we show that by checking only for the existence of a boundary path set, but not explicitly constructing one, we can solve the partial representation extension problem in linear time; see Section 5. Our algorithms use the above-mentioned algorithm of Kant and He [21] as a subroutine. We summarize our main contribution as follows.

Theorem 1. The partial representation extension problem for rectangular duals with a fixed regular edge labeling can be solved in linear time. For yes-instances, an explicit rectangular dual can be constructed within the same time bound.

\section{$2 \quad$ Linear Programming}

In this section, we describe how a rectangular dual of a given PTP graph and a given REL can be computed with the help of an LP. Felsner [14] used an LP to compute square duals, that is, a rectangular dual where each rectangle is actually a square. While Felsner's LP can be adapted to compute rectangular duals, we formulate our LP differently such that we can also use it for the partial representation extension problem and the simultaneous representation problem. For the same reason, our LP does not compete with the (linear-time) algorithm of Kant and He [21], which solves the task faster than our LP.

Our LP is a so-called system of difference constraints (SDC). This means that, if we write the LP in the standard form $A x \leq b$, every entry of the matrix $A$ is in $\{-1,0,1\}$ and in each row of $A$ at most one entry is a 1 and at most one entry is a -1 . The advantage of an SDC is that the Bellman-Ford algorithm can be used to find a solution (if one exists) in $\mathcal{O}\left(n^{2}+n m\right)$ time, where $n$ is the number of variables and $m$ is the number of constraints [11].

Let $G$ be a PTP graph, let $\left(L_{1}, L_{2}\right)$ be a REL of $G$, and let $\varepsilon>0$. We call our LP $\operatorname{RecDual}\left(G,\left(L_{1}, L_{2}\right), \varepsilon\right)$. We first describe the variables and then the constraints. We associate four variables with each vertex $u$ of $G$. The variables $x_{1, u}$ and $x_{2, u}$ denote the x-coordinates of the left side and the right side of $\mathcal{R}(u)$, respectively, and the variables $y_{1, u}$ and $y_{2, u}$ denote the y-coordinates of the bottom side and the top side of $\mathcal{R}(u)$, respectively. In what follows, we treat only the constraints regarding the $\mathrm{x}$-variables. The constraints regarding the $y$-variables are analogous. There are no constraints regarding both types of variables. We require that each rectangle has width and height at least $\varepsilon$, that is,

$$
x_{2, u}-x_{1, u} \geq \varepsilon \quad \text { for each vertex } u \text { of } G \text {. }
$$




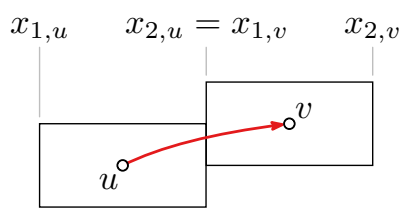

(a)

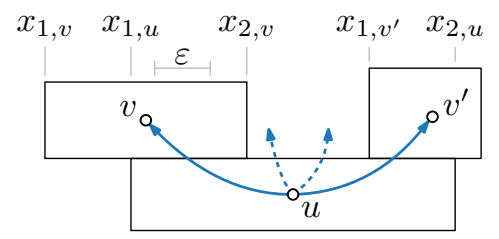

(b)

Fig. 3. The relation between variables of the SDC such that the edges of the REL are represented correctly.

We have two types of constraints for the edges.

First, for every edge $(u, v) \in E\left(L_{2}(G)\right)$, we ensure that the left side of $\mathcal{R}(v)$ touches the right side of $\mathcal{R}(u)$; see Fig. 3(a). In other words,

$$
x_{2, u}-x_{1, v}=0 \quad \text { for each edge }(u, v) \in E\left(L_{2}(G)\right) .
$$

(We treat the two edges $\left(v_{\mathrm{S}}, v_{\mathrm{W}}\right)$ and $\left(v_{\mathrm{E}}, v_{\mathrm{N}}\right)$ as edges of $L_{1}(G)$ and the two edges $\left(v_{\mathrm{W}}, v_{\mathrm{N}}\right)$ and $\left(v_{\mathrm{S}}, v_{\mathrm{E}}\right)$ as edges of $L_{2}(G)$; see Fig. 1. Thus, the lower left corner of the rectangular dual belongs to $\mathcal{R}\left(v_{\mathrm{S}}\right)$.)

Second, for every edge $(u, v) \in E\left(L_{1}(G)\right)$, we enforce that the rectangles $\mathcal{R}(u)$ and $\mathcal{R}(v)$ overlap horizontally; see Fig. 3(b). To this end, for a vertex $u$ in $G$, let $v$ and $v^{\prime}$ be the (clockwise) first and last outgoing neighbors of $u$ in $L_{1}(G)$. (They do not necessarily have to be distinct.) Then,

$$
\begin{aligned}
x_{2, v}-x_{1, u} \geq \varepsilon & \text { and } \\
x_{2, u}-x_{1, v^{\prime}} \geq \varepsilon & \text { for each vertex } u \text { of } G \text { with outgoing neighbors } v \text { and } v^{\prime} .
\end{aligned}
$$

As a result, $\mathcal{R}(v)$ and $\mathcal{R}\left(v^{\prime}\right)$ overlap with $\mathcal{R}(u)$ horizontally by at least $\varepsilon$. Rectangles corresponding to other outgoing neighbors of $u$ overlap with $\mathcal{R}(u)$ because they lie between $\mathcal{R}(v)$ and $\mathcal{R}\left(v^{\prime}\right)$ by the first type of constraint. We have analogous inequalities for the first and last incoming neighbor of $u$ in $L_{1}(G)$.

If the given graph $G$ has $n$ vertices, our SDC has $\mathcal{O}(n)$ variables and constraints, hence we can solve it in $\mathcal{O}\left(n^{2}\right)$ time using Bellman-Ford. This, however, tells us only whether a solution exists. In our case, this is not interesting since if we have a REL $\left(L_{1}, L_{2}\right)$ of $G$, then we know that a rectangular dual exists. We now show how to minimize the area and the perimeter of a rectangular dual, by reducing the optimization problem to the decision problem. (Kant and He [21] do not show this explicitly, but for a given REL, their linear-time algorithm yields a rectangular dual of minimum area and perimeter. They posed the open question whether it is possible to find a rectangular dual of minimum area or perimeter for a given PTP graph if the REL is not fixed. In any case, our slower LP is more flexible in that we can easily add additional constraints concerning, e.g., the size or position of rectangles.)

Note that the horizontal and vertical dimensions of a rectangular dual can be treated independently if the REL is fixed. Hence, it suffices to independently 
minimize the width and the height of the rectangular dual. If we set $x_{1, v_{\mathrm{S}}}=0$, then the x-coordinate $x_{2, v_{\mathrm{N}}}$ of the right side of $v_{\mathrm{N}}$ equals the width of the rectangular dual. Hence, we need to minimize $x_{2, v_{\mathrm{N}}}$. To this end, we do a binary search on $x_{2, v_{\mathrm{N}}}$ and solve the SDC for each value of $x_{2, v_{\mathrm{N}}}$ that the binary search considers. If we set $\varepsilon=1$, all constraints use integer values. Then the Bellman-Ford algorithm will yield a solution (that is, a rectangular dual) with integer coordinates. Note that the minimum width of such a rectangular dual is bounded by $n$ (since every integer between 0 and $n$ must be used by the x-coordinate of at least one left rectangle side and one right rectangle side). Hence, the binary search does $\mathcal{O}(\log n)$ steps, and we can solve the optimization problem in $\mathcal{O}\left(n^{2} \log n\right)$ time.

Partial Representation Extension. Let $\mathcal{P}$ be a partial rectangular dual of $G$. With a slight modification, we can use $\operatorname{RecDual}\left(G,\left(L_{1}, L_{2}\right), \varepsilon\right)$ to decide whether $\mathcal{P}$ admits a rectangular dual extension for $G$ and $\left(L_{1}, L_{2}\right)$. More precisely, for each fixed vertex $u$ of $\mathcal{P}$, we set $x_{1, u}, x_{2, u}, y_{1, u}$, and $y_{2, u}$ according to the fixed rectangle $\mathcal{R}(u)$ of $\mathcal{P}$. We call this LP $\operatorname{RepEx}\left(G,\left(L_{1}, L_{2}\right), \mathcal{P}, \varepsilon\right)$.

We need to set $\varepsilon$ such that rectangles can be placed between fixed rectangles with sizes and overlaps of at least $\varepsilon$. Let $X$ be the set of $\mathrm{x}$-coordinates $x_{1, u}$ and $x_{2, u}$ of all fixed vertices $u$. Define $Y$ analogously. Let $d$ be the minimum distance between any pair in $X$ or in $Y$. Observe that in a rectangular dual of $G$ at most $n$ distinct $\mathrm{x}$-coordinates are used for the $\mathrm{x}$-coordinates of the left and right sides of the rectangles. The analogous statement holds for the y-coordinates. Hence, we get the following lemma.

Lemma 2. If there exists any $\varepsilon>0$ such that $\operatorname{RepEx}\left(G,\left(L_{1}, L_{2}\right), \mathcal{P}, \varepsilon\right)$ has a solution, then $\operatorname{Rep} \operatorname{Ex}\left(G,\left(L_{1}, L_{2}\right), \mathcal{P}, d / n\right)$ has a solution as well.

The SDC $\operatorname{Rep} \operatorname{Ex}\left(G,\left(L_{1}, L_{2}\right), \mathcal{P}, d / n\right)$ for the partial rectangular dual extension problem has a worse asymptotic running time than the combinatorial methods of the subsequent sections. However, given $G,\left(L_{1}, L_{2}\right)$, and $\mathcal{P}$, the SDC is easy to generate and fast commercials solvers can be used. Furthermore, we can solve a slightly more general problem with the LP. For example, instead of specifying the position and the size of each fixed rectangle, we have the freedom to specify (or to bound) only some of these parameters.

Simultaneous Representation. Next, we describe how to use the SDC to solve the simultaneous rectangular dual representation problem when several graphs (sharing vertices) and their RELs are given. The idea is to generate one SDC per graph and then, for each vertex shared between two graphs, to identify the respective variables. Therefore, if two PTP graphs $G$ and $G^{\prime}$ share a vertex $u$, then the rectangles $\mathcal{R}(u)$ and $\mathcal{R}^{\prime}(u)$ in the rectangular duals of $G$ and $G^{\prime}$, respectively, will be identical.

More formally, let $G_{1}, \ldots, G_{k}$ be PTP graphs with RELs $\left(L_{1}, L_{2}\right)_{1}, \ldots$, $\left(L_{1}, L_{2}\right)_{k}$, respectively. For distinct $i$ and $j$ in $\{1, \ldots, k\}$, let $H_{i, j}$ be the common subgraph of $G_{i}$ and $G_{j}$. For each $i \in\{1, \ldots, k\}$, we generate the SDC 
$\operatorname{RepEx}\left(G_{i},\left(L_{1}, L_{2}\right)_{i}, 1\right)$ and let its variables have superscript $i$. We then merge the $k$ SDCs into a single SDC. To ensure a simultaneous representation, for each $u \in V\left(H_{i, j}\right)$, we set $x_{1, u}^{i}=x_{1, u}^{j}, x_{2, u}^{i}=x_{2, u}^{j}, y_{1, u}^{i}=y_{1, u}^{j}$, and $y_{2, u}^{i}=y_{2, u}^{j}$. The result is an SDC of size linear in the total number of vertices of $G_{1}, \ldots, G_{k}$. This yields the following theorem.

Theorem 3. The simultaneous representation problem for rectangular duals with a fixed regular edge labeling can be solved in quadratic time. For yes-instances, simultaneous rectangular duals can be constructed within the same time bound.

\section{Characterization}

In this section, we characterize when a given PTP graph $G$ with REL $\left(L_{1}, L_{2}\right)$, and partial rectangular dual $\mathcal{P}$ of $G$ admits an extension $\mathcal{R}$ that realizes $\left(L_{1}, L_{2}\right)$. Before we can explain our main idea, we require an observation and a few definitions.

We may assume that $v_{\mathrm{W}}, v_{\mathrm{S}}, v_{\mathrm{E}}$, and $v_{\mathrm{N}}$ are fixed vertices of $\mathcal{P}$. (Otherwise, we simply place the outer rectangles $\mathcal{P}\left(v_{\mathrm{W}}\right), \mathcal{P}\left(v_{\mathrm{S}}\right), \mathcal{P}\left(v_{\mathrm{E}}\right)$, and $\mathcal{P}\left(v_{\mathrm{N}}\right)$ appropriately around $\mathcal{P}$ such that they touch potential neighbours in $\mathcal{P}$.) The rectangles $\mathcal{P}\left(v_{\mathrm{W}}\right), \mathcal{P}\left(v_{\mathrm{S}}\right), \mathcal{P}\left(v_{\mathrm{E}}\right)$, and $\mathcal{P}\left(v_{\mathrm{N}}\right)$ thus form a frame with the area inside partially covered and partially uncovered. To make the question of whether this uncovered area can be filled with the rectangles of non-fixed vertices more accessible, we subdivide the uncovered area into smaller parts and then try to fill them one by one. More precisely and as illustrated in Fig. 4, we draw a vertical segment through the vertical sides of each fixed rectangle of an inner vertex until another fixed rectangle is hit. This divides the uncovered area inside the frame into (nonempty) vertical strips. We call the fixed rectangles bounding a vertical strip $S$ from below and above the start and end rectangles of $S$, respectively. We define horizontal strips symmetrically. The start and end rectangle of a horizontal strip are to its left and right, respectively.

The idea for our characterization is as follows. Consider an extension $\mathcal{R}$ of $\mathcal{P}$, where the strips are now filled with rectangles. The vertical strips thus naturally induce subgraphs in $L_{1}(G)$ containing the vertices that intersect it plus their

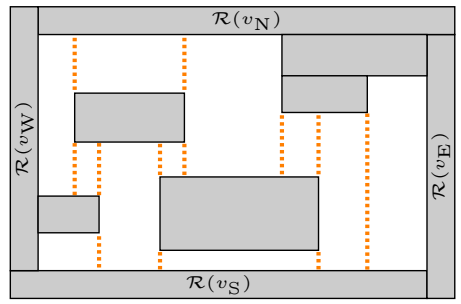

(a)

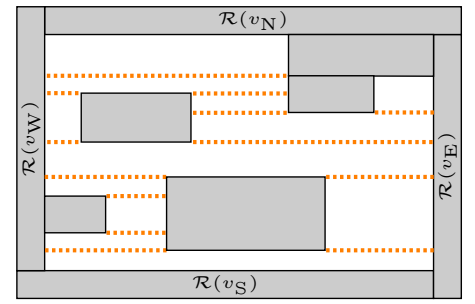

(b)

Fig. 4. Dissection of the interior of the frame into (a) vertical and (b) horizontal strips. 


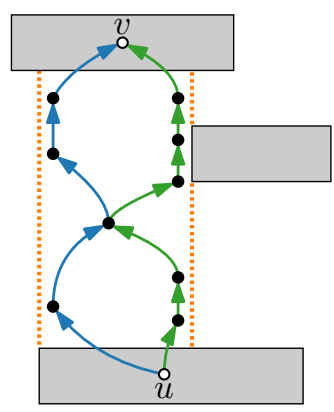

(a)

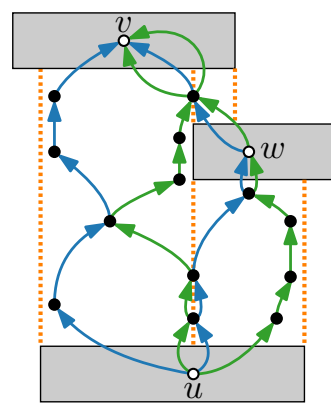

(b)

Fig. 5. (a) A boundary path pair for the strip with start rectangle $\mathcal{R}(u)$ and end rectangle $\mathcal{R}(v)$. (b) Neighboring strips can have overlapping boundary paths.

start and end rectangle. Together, these subgraphs cover the whole of $L_{1}(G)$. In particular, for a vertical strip $S$ with start rectangle $\mathcal{P}(u)$ and end rectangle $\mathcal{P}(v)$, the outer face of its induced subgraph consists of a path containing the rectangles along the left side of $S$ and a path along the right side of $S$. The idea is that, even with $\mathcal{R}$ not known, we have to be able to cover $L_{1}(G)$ (and $L_{2}(G)$ ) with subgraphs defined by pairs of boundary paths. We now make this precise.

For two paths $P$ and $P^{\prime}$ in $L_{1}(G)$, we write $P \preceq P^{\prime}$ if no vertex of $P$ lies to the right of $P^{\prime}$, i.e., there is no path from a vertex in $P^{\prime}$ to a vertex in $P \backslash P^{\prime}$ in $L_{2}(G)$. Let $S$ be a vertical strip with start rectangle $\mathcal{P}(u)$ and end rectangle $\mathcal{P}(v)$. A boundary path pair of $S$ is a pair of paths $\left\langle P_{1}(S), P_{\mathrm{r}}(S)\right\rangle$ from $u$ to $v$ in $L_{1}(G)$ such that $P_{1}(S) \preceq P_{\mathrm{r}}(S)$ and the only fixed vertices in $V\left(P_{1}(S) \cup P_{\mathrm{r}}(S)\right)$ are $u$ and $v$; see Fig. 5(a). Based on the boundary path pair of $S$, we define $S\left(L_{1}(G)\right)$ as the maximal subgraph of $L_{1}(G)$ that has precisely $P_{1}(S)$ and $P_{\mathrm{r}}(S)$ as the boundary of the outer face. The definitions for horizontal strips, where we order paths $P_{\mathrm{b}}(S)$ and $P_{\mathrm{t}}(S)$ from bottom to top, are analogous.

Let $\mathcal{S}_{1}$ and $\mathcal{S}_{2}$ be the sets of vertical and horizontal strips, respectively. We define a boundary path set of a REL $\left(L_{1}, L_{2}\right)$ as a set of boundary path pairs, one for each strip in $\mathcal{S}_{1}$ and $\mathcal{S}_{2}$, that satisfy the following properties (see Fig. $5(\mathrm{~b})$ ):

(B1) For strips $S$ and $S^{\prime}$ in $\mathcal{S}_{1}$ with $S$ left of $S^{\prime}$, it holds that $P_{\mathrm{r}}(S) \preceq P_{1}\left(S^{\prime}\right)$.

(B2) For strips $S$ and $S^{\prime}$ in $\mathcal{S}_{2}$ with $S$ below $S^{\prime}$, it holds that $P_{\mathrm{t}}(S) \preceq P_{\mathrm{b}}\left(S^{\prime}\right)$.

(B3) The vertical strips cover $L_{1}(G)$, and the horizontal strips cover $L_{2}(G)$, that is, $\bigcup_{S \in \mathcal{S}_{1}} S\left(L_{1}(G)\right)=L_{1}(G)$ and $\bigcup_{S \in \mathcal{S}_{2}} S\left(L_{2}(G)\right)=L_{2}(G)$.

Note that boundary paths of neighboring strips may overlap.

An extension $\mathcal{R}$ of $\mathcal{P}$ directly induces a boundary path set; for each, say, vertical strip we simply walk through the rectangles along its left and right boundary to find its boundary path pair. In the following, we show that the converse is also true. 


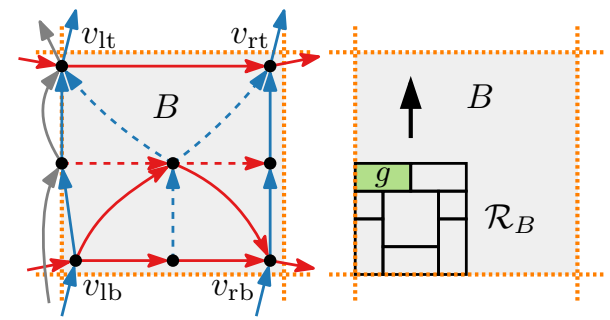

(a) (b)

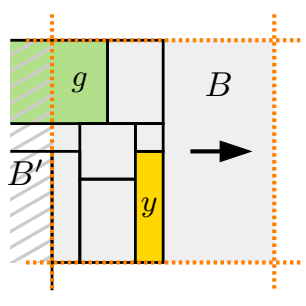

(c)

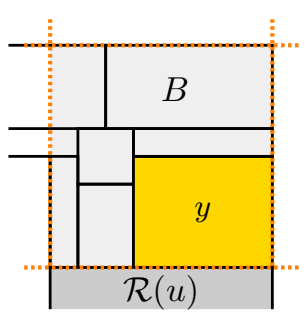

(d)

Fig. 6. (a) Graph $G_{B}$ for a box $B$; (b) representation $\mathcal{R}_{B}$ for $G_{B}$; (c) adjusting the left boundary of $\mathcal{R}_{B}$ to $\mathcal{R}_{B^{\prime}}$; and (d) adjusting the bottom boundary to $\mathcal{R}(u)$.

Theorem 4. Let $G$ be a PTP graph and let $\mathcal{P}$ be a partial rectangular dual of G. A REL $\left(L_{1}, L_{2}\right)$ of $G$ admits an extension of $\mathcal{P}$ if and only if $\left(L_{1}, L_{2}\right)$ admits a boundary path set.

Proof. Suppose that $\left(L_{1}, L_{2}\right)$ admits a boundary path set. We show how to use this set to construct an extension of $\mathcal{P}$.

Let $S$ be a vertical strip, let $S^{\prime}$ be a horizontal strip, and assume that $B=$ $S \cap S^{\prime}$ is nonempty. We call $B$ a box. All such boxes together with all fixed rectangles form a rectangle. We now fill the boxes from the bottom-left to the top-right.

The paths in the pairs $\left\langle P_{1}(S), P_{\mathrm{r}}(S)\right\rangle$ and $\left\langle P_{\mathrm{b}}\left(S^{\prime}\right), P_{\mathrm{t}}\left(S^{\prime}\right)\right\rangle$ pairwise intersect in single vertices $v_{\mathrm{lb}}, v_{\mathrm{lt}}, v_{\mathrm{rb}}, v_{\mathrm{rt}}$. Note that some or even all of these four vertices may coincide. Let $G_{B}$ be the subgraph of $G$ whose outer cycle is formed by the boundary path pairs between these vertices; see Fig. 6(a). If we enclose $G_{B}$ appropriately with a 4-cycle, we get a PTP graph with a REL and can apply the algorithm of Kant and He [21] to compute a rectangular dual $\mathcal{R}_{B}$ of $G_{B}$.

By the order in which we fill boxes, we have already treated those immediately to the left and below $B$; either of them may also be a fixed rectangle. Without loss of generality, we assume that there is a box $B^{\prime}$ that touches $B$ from the left and a fixed rectangle that touches $B$ from below.

First, we modify $\mathcal{R}_{B}$ such that it fits to the rectangular dual $\mathcal{R}_{B^{\prime}}$ that is drawn inside of $B^{\prime}$. Property (B1) of a boundary path set ensures that the rectangles in $\mathcal{R}_{B^{\prime}}$ that are adjacent to the right side of $\mathcal{R}_{B^{\prime}}$ are "compatible" to the rectangles in $\mathcal{R}_{B}$ that are adjacent to the left side of $\mathcal{R}_{B}$. Hence, starting with a tiny version of $\mathcal{R}_{B}$ placed in the lower left corner of $B$, we can stretch $\mathcal{R}_{B}$ vertically along suitable horizontal cuts such that, for every vertex $u$ in $V\left(G_{B^{\prime}}\right) \cap$ $V\left(G_{B}\right)$, the left piece of $\mathcal{R}(u)$ (in $B^{\prime}$ ) and the right piece of $\mathcal{R}(u)$ (in $B$ ) fit together; see the green rectangle $g$ in Fig. 6(b)-(c).

Now suppose that, for some fixed vertex $u$, the fixed rectangle $\mathcal{P}(u)$ bounds $B$ from below. Property (B3) of a boundary path set ensures that if we stretch $\mathcal{R}_{B}$ horizontally along some vertical cut, then we have the correct horizontal contacts with $\mathcal{P}(u)$; see the yellow rectangle $y$ in Fig. 6(c)-(d). 


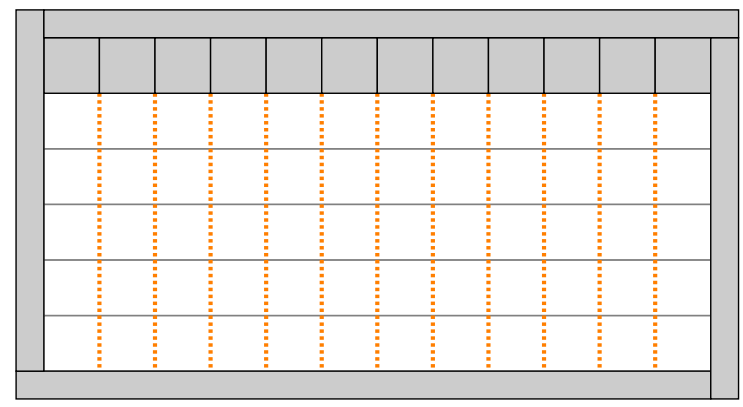

Fig. 7. A rectangular dual with a boundary path set of size $\Omega(n h)$ but only five unfixed vertices.

Finally, note that property (B3) ensures that, at the end of this construction, every vertex of $G$ is represented by a rectangle in $\mathcal{R}$.

Note that the existence of a boundary path set for a REL $\left(L_{1}, L_{2}\right)$ does not depend on the numeric values of the $\mathrm{x}$ - and $\mathrm{y}$-coordinates of the fixed rectangles in $\mathcal{P}$. In fact, we get the following corollary.

Corollary 5. Let $G$ be a PTP graph and let $\mathcal{P}$ be a partial rectangular dual of $G$. Whether a REL $\left(L_{1}, L_{2}\right)$ of $G$ admits an extension of $\mathcal{P}$ depends only on the order of the first and second $x$-coordinates and the order of the first and second $y$-coordinates of the fixed rectangles in $\mathcal{P}$.

We close this section with an observation about the potential size of boundary path sets. As we have noted above, a vertex may lie on multiple boundary paths; in fact, it may even lie on all of them as the example in Fig. 7 shows. Hence, the size of a boundary path set can be in $\Omega(n h)$, where $n=|V(G)|$ and $h=|\mathcal{P}|$.

\section{Finding a Boundary Path Set}

We now show how to compute a boundary path set for a given REL $\left(L_{1}, L_{2}\right)$ and a partial representation $\mathcal{P}$. The idea is as follows. As we did for the boxes in the proof of Theorem 4, we handle the vertical strips in $\mathcal{S}_{1}$ from bottom-left to topright. When computing the boundary path pair for a vertical strip $S \in \mathcal{S}_{1}$, we want the resulting graph $S\left(L_{1}(G)\right)$ to include all necessary vertices but otherwise as few vertices as possible. In particular, there may be rectangles that by $\left(L_{1}, L_{2}\right)$ need to have their left boundary align with the left boundary of $S$ and thus need to be in $P_{1}(S)$. To make this more precise, let $\mathcal{P}\left(v_{1}\right), \mathcal{P}\left(v_{2}\right), \ldots, \mathcal{P}\left(v_{k}\right)$ be the fixed rectangles whose right sides touch the left side of $S$. Let $u$ and $v$ be the fixed vertices corresponding to the start and end rectangle of $S$, respectively. Let $x$ be a vertex that lies on a path from $u$ to $v$ in $L_{1}(G)$. Then we say $x$ is left-bounded in $S$ if and only if one of the following conditions applies (see Fig. 8(a)): 
(L1) $x=u$ or $x=v$ and the left side of $\mathcal{P}(x)$ aligns with the left side of $S$;

(L2) $\left(v_{i}, x\right)$, for some $i \in\{1, \ldots, k\}$, is an edge in $L_{2}(G)$;

(L3) $(y, x)$ is the leftmost outgoing edge of $y$ and the leftmost incoming edge of $x$ in $L_{1}(G)$, and $y$ is left-bounded;

(L4) $(x, y)$ is the leftmost outgoing edge of $x$ and the leftmost incoming edge of $y$ in $L_{1}(G)$, and $y$ is left-bounded.

Condition (L2) applies if $\mathcal{R}(x)$ has to be directly to the right of a fixed rectangle left of $S$. Conditions (L3) and (L4) apply if the left side of $\mathcal{R}(x)$ has to align with the left side of a left-bounded rectangle $R(y)$ directly below or above, respectively. Note that in this case there exists also a vertex $y^{\prime}$ that is rightbounded in a strip $S^{\prime}$ left of $S$ and $\left(y^{\prime}, x\right) \in E\left(L_{2}(G)\right)$.

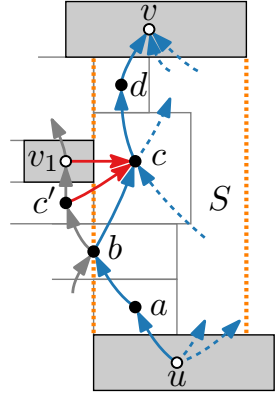

(a)

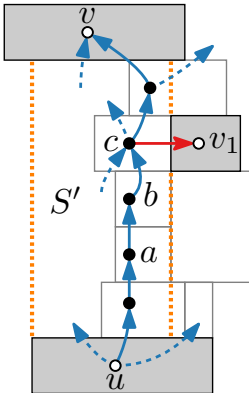

(b)

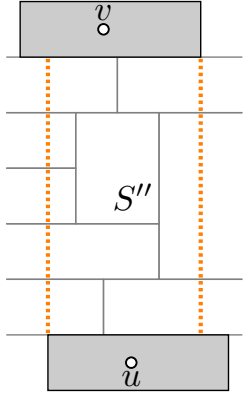

(c)

Fig. 8. (a) Vertex $u$ is left-bounded in $S$ by Condition (L1), $c$ on Condition (L2), a on Condition (L3), and $b$ is not left-bounded; (b) vertex $c$ is right-bounded in $S^{\prime}$ by Condition (R2), and $a$ and $b$ by Condition (L4); (c) $S^{\prime \prime}$ has neither left- nor rightbounded vertices except for $u$ and $v$.

Next, let $\mathcal{P}\left(v_{1}^{\prime}\right), \mathcal{P}\left(v_{2}^{\prime}\right), \ldots, \mathcal{P}\left(v_{k^{\prime}}^{\prime}\right)$ be the fixed rectangles whose left sides touch the right side of $S$. Then $x$ is right-bounded in $S$ if and only if one of the following conditions applies (see Fig. 8(b)):

(R1) $x=u$ or $x=v$ and the right side of $\mathcal{P}(x)$ aligns with the right side of $S$;

(R2) $\left(x, v_{i}^{\prime}\right)$, for some $i \in\left\{1, \ldots, k^{\prime}\right\}$, is an edge in $G_{2}$;

(R3) $(y, x)$ is the rightmost outgoing edge of $y$ and the rightmost incoming edge of $x$ in $L_{1}(G)$, and $y$ is right-bounded;

(R4) $(x, y)$ is the rightmost outgoing edge of $x$ and the rightmost incoming edge of $y$ in $L_{1}(G)$, and $y$ is right-bounded.

Note that $x$ can be both left- and right-bounded. Furthermore, starting from $u, v_{1}^{\prime}, \ldots, v_{k^{\prime}}^{\prime}, v$, these conditions can easily be checked for each strip. Overall, we can thus find all left- and right-bounded vertices of all strips in $\mathcal{O}(n)$ time.

Theorem 6. Let $G$ be a PTP graph with $n$ vertices and $R E L\left(L_{1}, L_{2}\right)$, let $\mathcal{P}$ be a partial rectangular dual of $G$, and let $h=|\mathcal{P}|$. 
In $\mathcal{O}(n h)$ time, we can decide whether $\left(L_{1}, L_{2}\right)$ admits a boundary path set with respect to $\mathcal{P}$ and, in the affirmative, compute it.

Proof. We show how to compute the boundary path pairs for vertical strips; horizontal strips can be treated analogously. Let $\mathcal{S}_{1}^{\checkmark}$ be the strips in $\mathcal{S}_{1}$ that have already been processed, that is, the strips for which the left and right boundary paths have already been computed. Let $S$ be a strip with start rectangle $\mathcal{P}(u)$ and end rectangle $\mathcal{P}(v)$ such that every strip left of $S$ is in $\mathcal{S}_{1}^{\checkmark}$.

An edge $(x, y)$ of $L_{1}(G)$ is suitable if one of the following conditions applies:

(E1) $y=v$;

(E2) $y$ is a non-fixed vertex in $P_{\mathrm{r}}\left(S^{\prime}\right)$, where $S^{\prime} \in \mathcal{S}_{1}^{\checkmark}$ is directly left of $S$ and $y$ is not right-bounded in $S^{\prime}$;

(E3) $y$ is a non-fixed vertex and $(x, y)$ is not an edge of $\mathcal{S}_{1}^{\checkmark}\left(L_{1}(G)\right)$.

Condition (E2) means that $\mathcal{R}(y)$ can span from $S^{\prime}$ into $S$ since it is not rightbounded in $S^{\prime}$. Thus, in Fig. 8(a) $(a, b)$ is suitable but $\left(b, c^{\prime}\right)$ is not. Furthermore, $(d, v)$ is suitable by Condition $(\mathrm{E} 1)$, and $(u, a),(a, b)$, and $(c, d)$ are suitable by Condition (E3). Note that $P_{1}(S)$ may only use suitable edges. Hence, to compute $P_{1}(S)$, we can start at $u$ and always add the leftmost suitable outgoing edge until we reach $v$. It follows that if, at some point, there is no suitable edge available, then $\left(L_{1}, L_{2}\right)$ does not admit a boundary path set. Taking the leftmost suitable outgoing edge ensures that $P_{1}(S)$ passes through all left-bounded vertices in $S$.

We now show how to construct $P_{\mathrm{r}}(S)$, enforcing that all right-bounded vertices lie on $P_{\mathrm{r}}(S)$. We thus start with the set of disjoint subpaths $P_{1}, P_{2}, \ldots, P_{k^{\prime}}$ induced by $u$, the right-bounded vertices, and $v$ ordered from bottom to top; see Fig. 9(a). Note that for a right-bounded vertex $x$ its rightmost outgoing edge also has to be in $P_{\mathrm{r}}(S)$, unless $x=v$, and its rightmost incoming edge also has to be in $P_{\mathrm{r}}(S)$, unless $x=u$. Therefore, we extend each subpath with these rightmost outgoing and incoming edges; see Fig. 9(b). For $i \in\left\{1, \ldots, k^{\prime}-1\right\}$, we then simultaneously extend $P_{i}$ and $P_{i+1}$ by always taking the leftmost suitable outgoing and incoming edge, respectively, but without crossing $P_{1}(S)$. If the extensions of $P_{i}$ and $P_{i+1}$ meet, we join them; see Fig. 9(c). Otherwise, both extensions will stop (due to a lack of suitable edges). In this case there is no path $P_{\mathrm{r}}(S)$, and then the REL $\left(L_{1}, L_{2}\right)$ does not admit a boundary path set.

Once $P_{1}(S)$ and $P_{\mathrm{r}}(S)$ have been computed successfully, we update the edge set of $\mathcal{S}_{1}^{\checkmark}\left(L_{1}(G)\right)$ before processing the next strip.

The runtime is linear in the size of the boundary path set, that is, $\mathcal{O}(n h)$.

Next, we show how to obtain an extension of $\mathcal{P}$ from a boundary path set.

Theorem 7. Let $G$ be a PTP graph with $n$ vertices and $R E L\left(L_{1}, L_{2}\right)$, let $\mathcal{P}$ be a partial rectangular dual of $G$, and let $h=|\mathcal{P}|$. Given a boundary path set of $\left(L_{1}, L_{2}\right)$, we can find an extension of $\mathcal{P}$ in $\mathcal{O}(n h)$ time.

Proof. In the proof of Theorem 4, we gave an algorithm that finds for every box $B$ obtained by the intersection of two strips the graph $G_{B}$ of vertices whose rectangles (partially) lie inside or on the boundary of $B$. The algorithm computes 


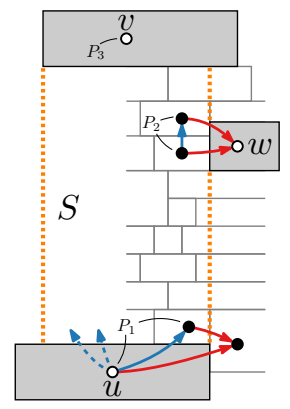

(a)

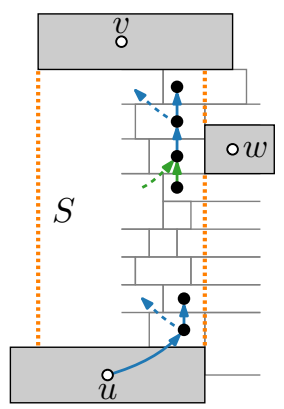

(b)

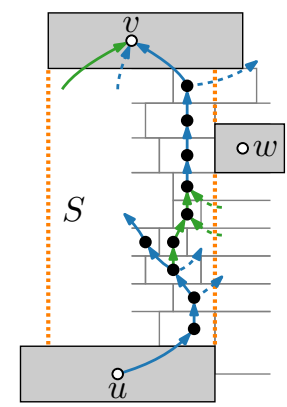

(c)

Fig. 9. Computation of $P_{\mathrm{r}}(S)$ (a) starting with subpaths induced by $u$, right-bounded vertices and $v$, (b) extending these subpaths with their rightmost predecessor and successor, and (c) leftwards until they meet. (Extensions downwards are shown green.)

a rectangular dual $\mathcal{R}_{B}$ of $G_{B}$ with the algorithm of Kant and He [21]. This requires $\mathcal{O}\left(\left|V\left(G_{B}\right)\right|\right)$ time per box [21]. Then $\mathcal{R}_{B}$ is fit into the extension built so far, which can also be done in $\mathcal{O}\left(\left|V\left(G_{B}\right)\right|\right)$ time per box.

We now argue that $\sum_{B}\left|V\left(G_{B}\right)\right|=\mathcal{O}(n h)$. Namely, a box $B$ either lies completely inside a rectangle, in which case $\left|V\left(G_{B}\right)\right|=1$, or it contains part of the boundary of every rectangle that corresponds to a vertex in $V\left(G_{B}\right)$. For any non-fixed vertex $v$, each of the four boundary sides of $\mathcal{R}(v)$ lies either inside a single strip or on the boundary between two strips. Hence, the boundary of $\mathcal{R}(v)$ can lie in only $\mathcal{O}(h)$ boxes in total. As there are $\mathcal{O}\left(h^{2}\right)$ boxes, we have $\sum_{B}\left|V\left(G_{B}\right)\right| \in \mathcal{O}\left(h^{2}+n h\right)=\mathcal{O}(n h)$.

\section{Linear-Time Algorithm}

Explicitly constructing a boundary path set, as in Theorem 6, requires time proportional in the size of the set, which can however be in $\Omega(n h)$. In this section, we show that even without an explicit construction, we can decide if a boundary path set exists, and if so, compute an extension. Both the decision and the computation can be done in linear time.

Our approach relies on the following observations. Suppose a boundary path set exists. Let $v$ be a non-fixed vertex that lies on a boundary path of vertical strips $S_{1}, \ldots, S_{k}$, ordered from left to right. Then the left boundary of $\mathcal{R}(v)$ lies in $S_{1}$ and the right boundary in $S_{k}$. Thus, to compute the x-coordinates of $\mathcal{R}(v)$, it suffices to know the leftmost and the rightmost boundary path on which $v$ lies. Instead of constructing all boundary path pairs of vertical strips, we only construct the subgraph $H_{1}$ of $L_{1}(G)$ induced by the fixed vertices and the vertices on the boundary path pairs. We call $H_{1}$ the vertical boundary graph of $\left(L_{1}, L_{2}\right)$. Furthermore, for each edge $e$ in $H_{1}$, we store the leftmost and the rightmost strip for which $e$ lies on a boundary path. Note that as $H_{1}$ is a subgraph of $L_{1}(G)$, the size of $H_{1}$ is in $\mathcal{O}(n)$. Analogously, we define $H_{2}$ for the horizontal strips. 
Before we show how to construct the boundary graphs $H_{1}$ and $H_{2}$, we prove that they suffice to compute an extension of $\mathcal{P}$.

Lemma 8. Let $G$ be a PTP graph with $n$ vertices and REL $\left(L_{1}, L_{2}\right)$, and let $\mathcal{P}$ be a partial rectangular dual of $G$. If boundary graphs $H_{1}$ and $H_{2}$ of $\left(L_{1}, L_{2}\right)$ are given, then an extension of $\mathcal{P}$ that realizes $\left(L_{1}, L_{2}\right)$ can be computed in $\mathcal{O}(n)$ time.

Proof. We show how to compute the x-coordinates of rectangles using $H_{1}$; the y-coordinates can be computed analogously with $H_{2}$. The idea is to compute a rectangular dual for each inner face of $H_{1}$, which in total will yield a full rectangular dual. Note that the boundary of each face of $H_{1}$ consists of two directed paths between a start and an end vertex. Therefore, each face has a single source, a single sink, a left path, and a right path.

We distinguish two types of inner faces of $H_{1}$, namely, those that contain part of the boundary of a strip and those that do not. A face $f$ of the former type can be identified by the occurrence of a right-bounded vertex $v$ on the left path of $f$ or a left-bounded vertex $v$ on the right path of $f$ where $v$ is not the source or sink of $f$; see Fig. 10(a). Note that in this case all inner vertices of the left path of $f$ are right-bounded and all inner vertices of the right path of $f$ are left-bounded. We then set the right x-coordinate of every inner vertex on the left path and the left $\mathrm{x}$-coordinate of every inner vertex on the right path to the $\mathrm{x}$-coordinate of the respective boundary of the strip.

Otherwise, an inner face $f$ of $H_{1}$ describes a region inside a strip of $S$; see Fig. 10(b). We define the graph $G_{f}$ as the subgraph of $G$ with all vertices that lie on or inside the cycle that is defined by $f$. By adding an outer four-cycle appropriately, we obtain a rectangular dual $\mathcal{R}_{f}$ of $G_{f}$ with the algorithm by Kant and He [21]. We then scale $\mathcal{R}_{f}$ to the width of $S$ and set the x-coordinates for the vertices in $G_{f}$ inside $S$ accordingly, that is, the right x-coordinate for the vertices on the left path of $f$, the left x-coordinate for vertices on the right path of $f$, and both x-coordinates for interior vertices of $G_{f}$.

After we have processed all faces, both $\mathrm{x}$-coordinates of all vertices are set since each vertex is either fixed, or has a face to the left and a face to the right, or lies inside a face. Since the faces are ordered from left to right in accordance with their respective strips, the computed $\mathrm{x}$-coordinates of the rectangles also form the correct horizontal adjacencies. We repeat this process with $H_{2}$ to compute the y-coordinates and thus to obtain also the correct vertical adjacencies.

Processing a face $f$ takes time linear in the size of $G_{f}$. Hence, the total running time is linear in the size of $L_{1}(G)$ and $L_{2}(G)$, and thus in $\mathcal{O}(n)$.

Lemma 9. Let $G$ be a PTP graph with $n$ vertices and $R E L\left(L_{1}, L_{2}\right)$, and let $\mathcal{P}$ be a partial rectangular dual of $G$. In $\mathcal{O}(n)$ time, we can decide whether $\left(L_{1}, L_{2}\right)$ admits a boundary path set with respect to $\mathcal{P}$ and, in the affirmative, compute boundary graphs $\mathrm{H}_{1}$ and $\mathrm{H}_{2}$.

Proof. As in the proof of Theorem 6, we focus again on vertical strips and process them from bottom-left to top-right. Let $\mathcal{S}_{1}^{\checkmark}$ be the strips in $\mathcal{S}_{1}$ that have already 


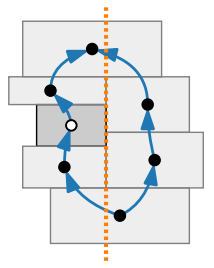

(a)

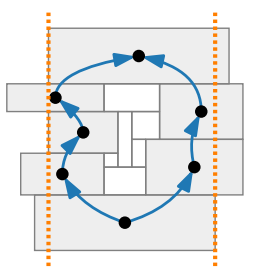

(b)

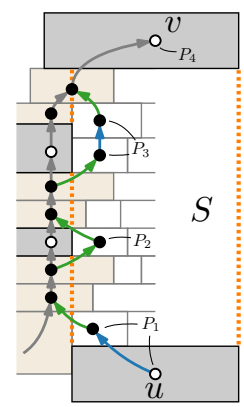

(c)

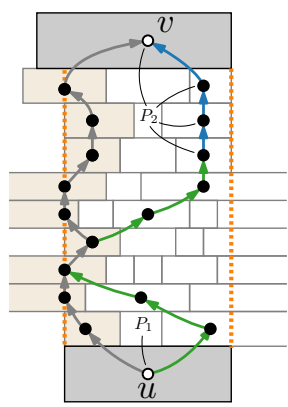

(d)

Fig. 10. Correspondence between a face of $H_{1}$ and (a) a part of the boundary of a strip or (b) a region inside a strip; extending $H_{1}$ with (c) $P_{1}(S)$ and (d) $P_{\mathrm{r}}(S)$.

been processed. Let $S$ be a strip with start rectangle $\mathcal{P}(u)$ and end rectangle $\mathcal{P}(v)$ such that every strip left of $S$ is in $\mathcal{S}_{1}^{\checkmark}$. The idea is to only compute the parts of $P_{1}(S)$ that do not coincide with a boundary path of a strip $S^{\prime} \in \mathcal{S}_{1}^{\checkmark}$ and the parts of $P_{\mathrm{r}}(S)$ that do not coincide with $P_{1}(S)$. Initially, set $H_{1}$ as the subgraph of $L_{1}(G)$ induced by the fixed vertices.

We start with $P_{1}(S)$; see Fig. 10(c). Observe that $P_{1}(S)$ should only consist of $u$, vertices left-bounded in $S, v$, and vertices on paths $P_{\mathrm{r}}\left(S^{\prime}\right)$ for $S^{\prime} \in \mathcal{S}_{1}^{\checkmark}$. Let $P_{1}, \ldots, P_{k}$ be the subpaths induced by $u$, vertices left-bounded in $S$, and $v$. Let $x_{i}$ be the first vertex of $P_{i}$ and $y_{i}$ the last. Further let $\left(w_{i}, x_{i}\right)$ be the leftmost incoming edge of $x_{i}$ and let $\left(y_{i}, z_{i}\right)$ be the leftmost outgoing edge of $y_{i}$. For $i \in\{2, \ldots, n\}, w_{i}$ already has to be in $H_{1}$ and may not be a fixed vertex; otherwise $P_{1}(S)$ does not exist. The analogous condition needs to hold for $\left(y_{i}, z_{i}\right)$. If this holds for each $i \in\{1, \ldots, k\}$, we add the vertices and edges in each $P_{i}$ as well as the edges $\left(w_{i}, x_{i}\right)$ and $\left(y_{i}, z_{i}\right)$ to $H_{1}$. Finally, we can test that the $P_{i}$ 's are in the correct order in $H_{1}$ with an st-ordering of $L_{1}(G)$.

Checking the existence of $P_{\mathrm{r}}(S)$ works like the construction in Theorem 6; see Fig. 10(d). Recall that we extended subpaths induced by $u$, right-bounded vertices, and $v$, first with rightmost incoming and outgoing edges appropriately, and then tried to join subsequent subpaths by taking the leftmost outgoing and incoming edges, respectively. Observe that reaching $P_{1}(S)$ during such an extension, now means that we encounter a non-fixed vertex in $H_{1}$; see Fig. 10 . Hence, here we stop extensions when we encounter a non-fixed vertex $v$ that is already in $H_{1}$. If connecting the subpaths to each other or $H_{1}$ is successful, we test their order again with an st-ordering of $L_{1}(G)$ and finally add them to $H_{1}$.

During the construction of $H_{1}$ we also need to label the faces with the strips they belong to. Therefore when a subpath $P_{i}$, for $i \in\{1, \ldots, k-1\}$, is added to $H_{1}$ as part of a left boundary path $P_{1}(S)$, we tell its left face that is lies on the left boundary of $S$. For any subpath added to $H_{1}$ as part of a right boundary path $P_{\mathrm{r}}(S)$, we tell its left face that it lies inside $S$.

Lastly, note that the running time is linear in the size of $H_{1}, H_{2}$ and $G$. 
As a result of Lemmas 8 and 9 we get our main result, Theorem 1 .

Theorem 1. The partial representation extension problem for rectangular duals with a fixed regular edge labeling can be solved in linear time. For yes-instances, an explicit rectangular dual can be constructed within the same time bound.

\section{Concluding Remarks}

In this paper, we have characterized the partial rectangular duals that admit an extension realizing a given REL in terms of boundary path sets. Based on this, we have given an algorithm that computes an extension, if it exists, in time proportional to the size of the boundary path set. We have sped up this algorithm by considering only the underlying simple graph of a boundary path set - the boundary graph.

We have also formulated a system of difference constraints (a special kind of LP) that can handle slightly more general versions of the partial rectangular dual extension problem. Furthermore, the LP can also be used to solve the simultaneous rectangular dual representation problem for PTP graphs with given RELs. One can simply formulate an LP for each graph separately and then concatenate them into a single LP where the variables for shared vertices are merged. As far as we know, this is the first result concerning the simultaneous representation of contact representations. It would be interesting to see this approach applied to other contact representations.

The partial rectangular dual extension problem remains open when no REL is specified. Eppstein et al. [13] gave algorithms that compute constrained areauniversal rectangular duals and solved the extension problem for RELs. A partial rectangular dual induces a partial REL. Hence an extension of a partial rectangular dual $\mathcal{P}$ can be found by computing every extension of this partial REL and by testing for each whether it admits an extension of $\mathcal{P}$, using our linear-time algorithm. There can, however, be exponentially many extensions of a partial REL. Naturally, we are interested in a faster approach.

\section{References}

1. Alam, J., Evans, W., Kobourov, S., Pupyrev, S., Toeniskoetter, J., Ueckerdt, T.: Contact representations of graphs in 3d. In: Dehne, F., Sack, J.R., Stege, U. (eds.) Algorithms and Data Structures (WADS). pp. 14-27. Springer (2015). doi: 10.1007/978-3-319-21840-3_2

2. Bläsius, T., Kobourov, S.G., Rutter, I.: Simultaneous embedding of planar graphs. In: Tamassia, R. (ed.) Handbook on Graph Drawing and Visualization, pp. 349381. Chapman and Hall/CRC (2013)

3. Bläsius, T., Rutter, I.: Simultaneous PQ-ordering with applications to constrained embedding problems. ACM Transactions on Algorithms 12(2), 16:1-16:46 (2016). doi:10.1145/2738054

4. Booth, K., Lueker, G.: Testing for the consecutive ones property, interval graphs, and graph planarity using PQ-tree algorithms. Journal of Computer and System Sciences 13(3), 335-379 (1976). doi:10.1016/S0022-0000(76)80045-1 
5. Brooks, R.L., Smith, C.A.B., Stone, A.H., Tutte, W.T.: The dissection of rectangles into squares. Duke Mathematical Journal 7(1), 312-340 (1940). doi:10.1215/S0012-7094-40-00718-9

6. Buchin, K., Speckmann, B., Verdonschot, S.: Evolution strategies for optimizing rectangular cartograms. In: Xiao, N., Kwan, M., Goodchild, M.F., Shekhar, S. (eds.) Geographic Information Science. Lecture Notes in Computer Science, vol. 7478, pp. 29-42. Springer (2012). doi:10.1007/978-3-642-33024-7_3

7. Buchsbaum, A.L., Gansner, E.R., Procopiuc, C.M., Venkatasubramanian, S.: Rectangular layouts and contact graphs. ACM Transactions on Algorithms 4(1) (2008). doi:10.1145/1328911.1328919

8. Chaplick, S., Dorbec, P., Kratochvíl, J., Montassier, M., Stacho, J.: Contact representations of planar graphs: Extending a partial representation is hard. In: Kratsch, D., Todinca, I. (eds.) Graph-Theoretic Concepts in Computer Science (WG). pp. 139-151. Springer (2014). doi:10.1007/978-3-319-12340-0_12

9. Chaplick, S., Fulek, R., Klavík, P.: Extending partial representations of circle graphs. Journal of Graph Theory 91(4), 365-394 (2019). doi:10.1002/jgt.22436

10. Chaplick, S., Guspiel, G., Gutowski, G., Krawczyk, T., Liotta, G.: The partial visibility representation extension problem. Algorithmica 80(8), 2286-2323 (2018). doi:10.1007/s00453-017-0322-4

11. Cormen, T.H., Leiserson, C.E., Rivest, R.L., Stein, C.: Introduction to Algorithms. MIT Press, 3rd edn. (2009), https://mitpress.mit.edu/books/ introduction-algorithms-third-edition

12. Duncan, C.A., Gansner, E.R., Hu, Y., Kaufmann, M., Kobourov, S.G.: Optimal polygonal representation of planar graphs. Algorithmica 63(3), 672-691 (2012). doi:10.1007/s00453-011-9525-2

13. Eppstein, D., Mumford, E., Speckmann, B., Verbeek, K.: Area-universal and constrained rectangular layouts. SIAM Journal on Computing 41(3), 537-564 (2012). doi:10.1137/110834032

14. Felsner, S.: Rectangle and square representations of planar graphs. In: Pach, J. (ed.) Thirty Essays on Geometric Graph Theory, pp. 213-248. Springer (2013). doi : 10.1007/978-1-4614-0110-0_12

15. de Fraysseix, H., de Mendez, P.O., Rosenstiehl, P.: On triangle contact graphs. Combinatorics, Probability and Computing 3(2), 233-246 (1994). doi:10.1017/S0963548300001139

16. Gabriel, K.R., Sokal, R.R.: A new statistical approach to geographic variation analysis. Systematic Biology 18(3), 259-278 (1969). doi:10.2307/2412323

17. Gonçalves, D., Lévêque, B., Pinlou, A.: Triangle contact representations and duality. Discrete \& Computational Geometry 48(1), 239-254 (2012). doi:10.1007/978-3-642-18469-7_24

18. Heilmann, R., Keim, D.A., Panse, C., Sips, M.: RecMap: Rectangular map approximations. In: Ward, M.O., Munzner, T. (eds.) Information Visualization (InfoVis). pp. 33-40. IEEE (2004). doi:10.1109/INFVIS.2004.57

19. Jampani, K.R., Lubiw, A.: Simultaneous interval graphs. In: Cheong, O., Chwa, K.Y., Park, K. (eds.) Algorithms and Computation (ISAAC), Part I. Lecture Notes in Computer Science, vol. 6506, pp. 206-217. Springer (2010). doi : 10.1007/978-3-642-17517-6_20

20. Jampani, K.R., Lubiw, A.: The simultaneous representation problem for chordal, comparability and permutation graphs. Journal of Graph Algorithms and Applications 16(2), 283-315 (2012). doi:10.7155/jgaa.00259 
21. Kant, G., He, X.: Regular edge labeling of 4-connected plane graphs and its applications in graph drawing problems. Theoretical Computer Science 172(1), 175-193 (1997). doi:10.1016/S0304-3975(95)00257-X

22. Klavík, P., Kratochvíl, J., Otachi, Y., Rutter, I., Saitoh, T., Saumell, M., Vyskočil, T.: Extending partial representations of proper and unit interval graphs. Algorithmica 77(4), 1071-1104 (2017). doi:10.1007/s00453-016-0133-z

23. Klavík, P., Kratochvíl, J., Otachi, Y., Saitoh, T., Vyskočil, T.: Extending partial representations of interval graphs. Algorithmica 78(3), 945-967 (2017). doi:10.1007/s00453-016-0186-z

24. Klawitter, J., Nöllenburg, M., Ueckerdt, T.: Combinatorial properties of trianglefree rectangle arrangements and the squarability problem. In: Di Giacomo, E., Lubiw, A. (eds.) Graph Drawing and Network Visualization (GD). pp. 231-244. Springer (2015). doi:10.1007/978-3-319-27261-0_20

25. Koebe, P.: Kontaktprobleme der konformen Abbildung. Berichte über die Verhandlungen der Sächsischen Akademie der Wissenschaften zu Leipzig. Math.-Phys. Klasse 88 pp. 141-164 (1936)

26. Koźmiński, K., Kinnen, E.: Rectangular duals of planar graphs. Networks 15(2), 145-157 (1985). doi:10.1002/net.3230150202

27. Krawczyk, T., Walczak, B.: Extending partial representations of trapezoid graphs. In: Bodlaender, H.L., Woeginger, G.J. (eds.) Graph-Theoretic Concepts in Computer Science (WG). pp. 358-371. Springer (2017). doi:10.1007/978-3-319-68705-6_27

28. van Kreveld, M.J., Speckmann, B.: On rectangular cartograms. Computational Geometry 37(3), 175-187 (2007). doi:10.1016/j.comgeo.2006.06.002

29. Kusters, V., Speckmann, B.: Towards characterizing graphs with a sliceable rectangular dual. In: Di Giacomo, E., Lubiw, A. (eds.) Graph Drawing and Network Visualization (GD). pp. 460-471. Springer (2015). doi: 10.1007/978-3-319-27261-0_38

30. Leinwand, S.M., Yen-Tai Lai: An algorithm for building rectangular floorplans. In: Design Automation Conference (DAC). pp. 663-664 (1984). doi:10.1109/DAC.1984.1585874

31. Nusrat, S., Kobourov, S.G.: The state of the art in cartograms. Computer Graphics Forum 35(3), 619-642 (2016). doi:10.1111/cgf .12932

32. Raisz, E.: The rectangular statistical cartogram. Geographical Review 24(2), 292 296 (1934). doi: 10.2307/208794

33. Rutter, I.: Simultaneous embedding. In: Hong, S.H., Tokuyama, T. (eds.) Beyond Planar Graphs: Communications of NII Shonan Meetings, pp. 237-265. Springer Singapore (2020). doi:10.1007/978-981-15-6533-5_13

34. Rutter, I., Strash, D., Stumpf, P., Vollmer, M.: Simultaneous representation of proper and unit interval graphs. In: Bender, M.A., Svensson, O., Herman, G. (eds.) European Symposium on Algorithms (ESA). LIPIcs, vol. 144, pp. 80:1-80:15. Schloss Dagstuhl - Leibniz-Zentrum für Informatik (2019). doi : 10.4230/LIPIcs.ESA . 2019.80

35. Steadman, P.: Graph theoretic representation of architectural arrangement. Architectural Research and Teaching pp. 161-172 (1973)

36. Yeap, G.K.H., Sarrafzadeh, M.: Sliceable floorplanning by graph dualization. SIAM Journal on Discrete Mathematics 8(2), 258-280 (1995). doi:10.1137/S0895480191266700 